\title{
Deciding when to stop: towards evidence-based deprescribing of drugs in older populations
}

\section{Ian A Scott, ${ }^{1}$ Leonard C Gray, ${ }^{2}$ Jennifer H Martin, ${ }^{3}$ Peter I Pillans, ${ }^{4}$ Charles A Mitchell ${ }^{5}$}

$10.1136 /$ eb-2012-100930

${ }^{1}$ Department of Internal

Medicine and Clinical

Epidemiology, University of

Queensland, Princess Alexandra

Hospital, Brisbane, Queensland,

Australia

${ }^{2}$ Centre for Academic Research

in Geriatric Medicine, University

of Queensland, Princess

Alexandra Hospital, Brisbane,

Queensland, Australia

${ }^{3}$ Southern Discipline of

Medicine School of Medicine,

University of Queensland,

Princess Alexandra Hospital,

Brisbane, Queensland, Australia

${ }^{4}$ Department of Clinical

Pharmacology, Princess

Alexandra Hospital, Brisbane,

Queensland, Australia.

${ }^{5}$ Centre for Safe and Effective

Prescribing, Pharmaceuticals

Australia Centre of Excellence,

University of Queensland,

Brisbane, Queensland, Australia

\section{Correspondence to:}

Dr lan A Scott,

Department of Internal Medicine and Clinical Epidemiology, University of Queensland, Level 5A, Princess Alexandra Hospital, Ipswich Road, Brisbane QLD 4102, Australia;

ian_scott@health.qld.gov.au

\section{Abstract}

Minimising the harm from inappropriate prescribing in older populations is a major urgent concern for modern healthcare systems. In everyday encounters between prescribers and patients, opportunities should be taken to identify patients at high risk of harm from polypharmacy and reappraise their need for specific drugs. Attempts to reconcile life expectancy, comorbidity burden, care goals and patient preferences with the benefits and harms of medications should be made in every patient at significant risk. Drugs identified by this process of reconciliation as conferring little or no benefit and/or excessive risk of harm should be candidates for discontinuation. Evidence supporting a structured approach to drug discontinuation (or deprescribing) is emerging, and while many barriers to deprescribing exist in routine practice, various enabling strategies can help overcome them.

\section{Introduction}

Minimising the harm of inappropriate prescribing in older populations (persons aged 65 years or more) is a major urgent concern for modern healthcare systems. Over a 5-year period, one in four older persons are hospitalised for medication-related problems, ${ }^{1}$ accounting for more than $10 \%$ of all hospital admissions in this population, ${ }^{2}$ with between $30 \%$ and 55\% deemed preventable. ${ }^{3}{ }^{4}$ In the wider community, one in three subjects receiving five or more drugs suffer an adverse drug reaction (ADR) every 12 months, with more than a quarter deemed preventable. ${ }^{5}$ Up to $18 \%$ of all inpatient deaths are attributable in part to $\mathrm{ADRs}^{6}$ and $44 \%$ of patients at hospital discharge are prescribed at least one unnecessary drug. $^{7}$

The drivers of polypharmacy are multiple: plethora of disease-specific clinical guideline recommendations (many of which may not be applicable to older patients with multiple comorbidities) coupled with guidelinederived quality indicators and performance incentives; patient and carer expectations and provider sensitivity about age discrimination; inadequate knowledge of geriatric therapeutics and toxicology; focus on treating acute disease (often with additional drugs) while neglecting reappraisal of existing drugs for chronic disease; and ADRs being misinterpreted as new diseases requiring more drugs. Clinical research, guidelines and models of care seldom support the complex and difficult decisions about when to stop existing drugs or withhold new ones in older patients with multiple comorbidities. ${ }^{8}$

\section{Issues to consider when discontinuing inappropriate medications}

Appropriate prescribing comprises use of drugs associated with strong evidence of benefit while eliminating medications with questionable or no evidence of efficacy, unfavourable risk-benefit trade-offs or expressed patient preference to avoid. We have formulated an evidencebased 10-step discontinuation guide based on a conceptual framework fully described elsewhere (see figure 1). ${ }^{9}$ Face validity of this guide has been confirmed among an unselected group of doctors whose prescribing intentions with regard to a hypothetical case changed significantly after guide application. ${ }^{10}$ Certain issues relating to this framework deserve particular emphasis.

All medications a patient is taking, including complementary and non-prescription drugs, must be accurately ascertained. Limited evidence suggests the "brown paper bag' method of asking patients (or carers) to physically bring all medicines with them to the clinical encounter is the best way to reconcile medications. Asking specific questions to detect non-adherence also helps identify drugs which may be ceased.

Patients at highest risk of ADRs are those prescribed the greatest number of drugs (up to $82 \%$ risk with seven drugs or more ${ }^{11}$ ) or which include high-risk drugs (antithrombotic agents, insulin, oral hypoglycaemic agents, cardiovascular and central nervous system drugs). Clinicians need to look for pre-existing drug toxicity presenting as falls, confusion and lethargy, effects too often inappropriately attributed to ageing.

Estimating a patient's life expectancy is important in determining the goals of care and potential long-term value of many preventive medications. Patients with a limited lifespan (12 months or less) due to marked frailty, advanced dementia, metastatic cancer and endstage organ disease should have more conservative care goals and their preferences may call for reductions in drug burden. In particular, such patients will gain little value from medications that take more than a year for benefits to manifest, such as bisphosphonate therapy (to prevent osteoporotic fractures) or statins (to prevent cardiovascular events).

Even when medications are intended to treat current active disease, diagnoses should be verified as symptoms and signs may manifest in an unusual manner in older people. Where diseases have been misdiagnosed or are no longer active, attendant drugs should be discontinued. Cross-sectional studies suggest diagnoses of heart failure, Parkinson's disease and depression, while prevalent in older populations, often lack substantiation in individual cases. ${ }^{12} 13$

Even when clear indications exist, recent studies have challenged the safety in older patients of aggressive 'treat-to-target' drug regimens for diseases such as diabetes and hypertension. Absolute disease risk must be reconciled with absolute risk of harm using tools such as the CHADS2 score for estimating thromboembolic stroke risk and HAS-BLED score for estimating 
1. Accurately ascertain all current drug use

- 'brown, paper bag' medication reconciliation

2. Identify patients at risk of, or suffering, ADR

- at risk: $\geq 8$ medications advanced age ( $>75$ years) high-risk medications

- assess for current, past or highly likely future toxicity

3. Estimate life expectancy

- clinical, prognostication tools or lifespan calculators

4. Define overall care goals

- consider current functional status and quality of life with Ireference to estimated life expectancy

5. Verify current indications for ongoing treatments

- perform diagnosis-medication reconciliation

- confirm diagnostic labels against formal diagnostic criteria

- ascertain, for each confirmed diagnosis, drug appropriateness

6 .

- estimate clinical impact and time to future treatment benefit

- compare this estimate with expected lifespan

7. Determine absolute benefit-harm thresholds of medications

- reconcile estimates of absolute benefit and harm using prediction tools (see http://www.mdcalc.com)

8. Review the relative utility of individual drugs

- rank drugs according to the relative utility from high to low based on predicted benefit, harm, administration and monitoring burden I

9. Identify drugs to be discontinued and seek patient consent

- reconcile drugs for discontinuation with patient preferences

$\checkmark$

10. Devise and implement drug discontinuation plan with close monitoring

Figure 1 Tool for identifying and discontinuing potentially inappropriate drugs.

risk of major bleeding from anticoagulants in patients with non-valvular atrial fibrillation.

In the final evaluation, the overall utility of individual drugs spans a continuum between those of high utility (proven and sizeable benefit in virtually all eligible older patients, little administration or monitoring burden, small potential for harm) and those of low utility (questionable indications, onerous administration or monitoring burden, high risk of harm in most, if not all, patients). In between are drugs whose relative utility rests on a case-by-case consideration of the framework steps in figure 1 and the questions listed in Table 1. Validated screening tools such as the updated Beers criteria ${ }^{14}$ or STOPP tool ${ }^{15}$ can also identify many (although by no means all) low-utility drugs.

The final say needs to rest with the patient or, where necessary, his/her delegated advocate. Even for seemingly effective drugs with valid indications and little or no risk of serious harm, older patients place as much, if not more, emphasis on avoiding short-term toxicity affecting cognitive, physical and emotional functioneven if uncommon-than on primary effects on future disease risk. ${ }^{16}$ The more drugs prescribed and the greater the potential for more side-effects, the higher the rates of non-adherence-as high as $85 \% .{ }^{17}$ Once a discontinuation regimen has been decided, selected drugs can then be ceased or weaned, one at a time, while monitoring the patient closely for disease recrudescence or onset of withdrawal or rebound syndromes. ${ }^{7}$ Table 2 lists drugs which warrant gradual weaning over extended periods of time.

\section{Evidence for drug discontinuation}

Evidence is emerging for more proactive approaches to drug discontinuation. In one trial involving 119 older disabled patients, 332 different drugs (average 2.8 drugs per patient) were discontinued using an algorithm, leading to reductions in 12 month mortality (21\% vs $45 \%$ ), referral rates to acute care facilities ( $12 \%$ vs 30\%) and drug costs. ${ }^{18}$ In another study using the same algorithm and involving 70 community-dwelling older patients prescribed a mean of 7.7 drugs, 58\% of medications were discontinued with an $81 \%$ success rate with no long-term adverse consequences and almost $90 \%$ of patients reporting global improvement in health. ${ }^{19}$ Another study used Medicare claims data in the USA to retrospectively identify patients prescribed inappropriate drugs, following which prescribers were mailed cautionary information which led, 6 months later, to discontinuation of $49 \%$ of these drugs. ${ }^{20}$ In other cohort studies, more than $50 \%$ of drugs prescribed to older patients have been discontinued or reduced in dose without major untoward effects. ${ }^{21}$ In a study of 119 nursing home residents receiving selective serotonin reuptake inhibitors for more than 12 months, withdrawal was successfully achieved in 52\% following a structured drug review. ${ }^{22}$

A randomised trial showed significant decrease in numbers of drugs and trend towards fewer falls in patients whose medications were reviewed by pharmacists using STOPP criteria. $^{23}$ In one systematic review, between $20 \%$ and $85 \%$ of patients remained normotensive without any increase in mortality after withdrawal 
Table 1 How to determine drug utility

What is the strength of indication (in order of decreasing utility)?

The drug:

1. Provides immediate relief of distressing symptoms (eg, analgesics, antiemetics and antipruritics).

2. Effectively modifies an acute condition that is life threatening or will soon result in distressing symptoms if not treated (eg, antibiotics for sepsis, diuretics for acute heart failure and bronchodilators for asthma).

3. Effectively modifies a chronic condition that might progress to become life threatening or cause distressing symptoms if not treated (eg, methotrexate for rheumatoid arthritis and ACE inhibitors for chronic heart failure).

4. Has the potential to prevent serious diseases or adverse events in the future, without immediate effect (eg, antiplatelet agents to prevent cardiovascular disease, bisphosphonates to prevent osteoporotic fractures and antihypertensives to prevent stroke).

5. Is unlikely to be useful in either the short or long term (eg, vitamin supplements).

6. Is prescribed where a non-drug therapy (eg, physiotherapy instead of non-steroidal anti-inflammatory drugs for lower back pain) is more beneficial.

What is the likelihood of misuse, toxicity or non-adherence?

The drug:

1. Is associated with little benefit and high risk of toxicity in most older patients (on the basis of Beers' criteria or other drugs-to-avoid lists).

2. Is a duplication in drug therapy (ie, a second drug from the same class).

3. Is prescribed for an adverse drug reaction (ADR).

If so, can the drug causing the ADR be withdrawn or substituted with another agent less likely to cause an ADR?

4. Is a potentially beneficial drug but is prescribed at a dose likely to cause toxicity.

If so, can another effective, less toxic dose or medication be substituted?

5. Has the potential for significant drug-drug or drug-disease interactions.

If so, can it be withdrawn or substituted with another agent less likely to cause an interaction?

6. Is taken more often than once daily.

Can another equally effective drug be substituted that can be scheduled once daily?

7. Can be safely administered as a combination medication.

8. Is causing significant difficulties with adherence?

If so, can it be withdrawn or substituted with another agent more likely to be taken?

of antihypertensive drugs, ${ }^{24}$ while in another review, discontinuation of psychotropic medications led to fewer falls and improvement in daily function and cognition. ${ }^{25}$ Other systematic reviews of deprescribing trials ${ }^{26-28}$ describe successful results arising from medication reviews by clinical pharmacists, prescriber

Table 2 Medications commonly associated with discontinuation syndromes which require slow weaning

\begin{tabular}{|c|c|c|}
\hline Medication & $\begin{array}{l}\text { Type of discontinuation } \\
\text { syndrome }\end{array}$ & Clinical manifestations \\
\hline$\alpha$-Blockers & W, R & Agitation, headache, hypertension and palpitations \\
\hline ACE-inhibitors & $\mathrm{D}$ & Heart failure and hypertension \\
\hline Antianginal agents & $\mathrm{D}$ & Angina \\
\hline Anticonvulsants & W, D & Anxiety, depression and seizures \\
\hline Antidepressants & W, D & $\begin{array}{l}\text { Akathisia, anxiety, chills, coryza, gastrointestinal distress, headache, } \\
\text { insomnia, irritability, malaise, myalgia and depression }\end{array}$ \\
\hline $\begin{array}{l}\text { Antiparkinsonian } \\
\text { agents }\end{array}$ & W, D, R & Hypotension, psychosis, pulmonary embolism, rigidity and tremor \\
\hline Antipsychotic & w & Dyskinesias, insomnia, nausea and restlessness \\
\hline Anticholinergics & w & Anxiety, nausea, vomiting, headache and dizziness \\
\hline Baclofen & W, R & $\begin{array}{l}\text { Agitation, anxiety, confusion, depression, hallucinations, hypertonia, } \\
\text { insomnia, mania, nightmares, paranoia and seizures }\end{array}$ \\
\hline Benzodiazepines & w & Agitation, anxiety, confusion, delirium, insomnia and seizures \\
\hline$\beta$-Blockers & W, D & Angina, anxiety, hypertension, acute coronary syndrome and tachycardia \\
\hline Corticosteroid & $W, R, D$ & $\begin{array}{l}\text { Anorexia, hypotension, nausea, weakness, hypothalamic-pituitary- } \\
\text { adrenal axis suppression and inflammatory states }\end{array}$ \\
\hline Digoxin & D & Heart failure and palpitations \\
\hline Diuretic & D & Heart failure and hypertension \\
\hline Narcotic analgesia & w & $\begin{array}{l}\text { Abdominal cramping, anger, anxiety, chills, diaphoresis, diarrhoea, } \\
\text { insomnia and restlessness }\end{array}$ \\
\hline NSAIDs & $\mathrm{D}$ & Recurrence of gout and arthritis \\
\hline
\end{tabular}

$D$, disease recrudescence; NSAID, non-steroidal anti-inflammatory drug; $R$, rebound; $W$, withdrawal. 
education programmes and reminders, academic detailing, comprehensive geriatric assessments, multidisciplinary conferences and patient-centred interventions.

\section{Conclusion}

Evidence is accumulating on the need for, and feasibility and benefits of, appropriate drug discontinuation in older patients. The systematic approach described here provides guidance in performing this increasingly important task and also serves as a checklist when considering start of additional drugs. While ceasing medications can be difficult and time consuming, prescribers have a responsibility to minimise the potential for harm and waste of resources arising from inappropriate polypharmacy in vulnerable older persons.

Contributors The authors undertook all research for this article and had access to all data. IAS conceived the idea for the article and wrote the first draft; LCG, JHM, PIP and CAM critically reviewed the manuscript, suggested amendments and provided additional references.

Competing interests All authors except IAS declared that the answer to the questions on the competing interest form are all NO and therefore have nothing to declare. IAS has declared he was a recipient of a grant from the Princess Alexandra Hospital Private Practice Research Fund.

Guarantor IAS as the principal investigator had full access to all of the data in the study and takes responsibility for the integrity of the data and the accuracy of the data analysis.

\section{References}

1. Kalisch LM, Caughey GE, Barratt JD, et al. Prevalence of preventable medication-related hospitalizations in Australia: an opportunity to reduce harm. Int J Qual Health Care 2012;24:239-49.

2. Kongkaew C, Noyce PR, Ashcroft DM. Hospital admissions associated with adverse drug reactions: a systematic review of prospective observational studies. Ann Pharmacother 2008;42:1017-25.

3. Doucet J, Jego A, Noel D, et al. Preventable and non-preventable risk factors for adverse drug events related to hospital admissions in the elderly. A prospective study. Clin Drug Invest 2002;22:385-92.

4. Chan M, Nicklason F, Vial JH. Adverse drug events as a cause of hospital admission in the elderly. Int Med J 2001;31:199-205.

5. Hanlon JT, Schmader KE, Koronkowski MJ, et al. Adverse drug events in high risk older outpatients. J Am Geriatr Soc 1997;45:945-8.

6. Buajordet I, Ebbesen J, Erikssen J, et al. Fatal adverse drug events: the paradox of drug treatment. J Intern Med 2001;250:327-41.

7. Hajjar ER, Hanlon JT, Sloane RJ, et al. Unnecessary drug use in frail older people at hospital discharge. $J$ Am Geriatr Soc 2005;53:1518-23.

8. Mangin D, Heath I, Jamoulle M. Beyond diagnosis: rising to the multimorbidity challenge. BMJ 2012;344:e3526.
9. Scott IA, Martin JH, Gray LA, et al. Minimising inappropriate medication use in older populations-a 10 step conceptual framework. Am J Med 2012;125:529-37.

10. Scott IA, Martin JH, Gray LA, et al. Effects of a drug minimisation guide on prescribing intentions in elderly persons with polypharmacy. Drugs Ageing 2012;29:659-67.

11. Goldberg RM, Mabee J, Chan L, et al. Drug-drug and drug-disease interactions in the emergency department: analysis of a high risk population. Am J Emerg Med 1996;14:447-50.

12. Owen A, Cox S. Diagnosis of heart failure in elderly patients in primary care. Eur J Heart Fail 2001;3:79-81.

13. Meara J, Bhowmick BK, Hobson P. Accuracy of diagnosis in patients with presumed Parkinson's disease. Age Ageing 1999;28:99-102.

14. The American Geriatrics Society 2012 Beers Criteria Update Expert Panel. American Geriatrics Society updated criteria for potentially inappropriate medication use in older adults. $J \mathrm{Am}$ Geriatr Soc 2012;60:616-31.

15. Gallagher P, Ryan C, Byrne S, et al. STOPP (Screening Tool of Older Persons' Prescriptions) and START (Screening Tool to Alert doctors to Right Treatment). Consensus validation. Int J Clin Pharmacol Ther 2008;46:72-83.

16. Fried TR, Tinetti ME, Towle V, et al. Effects of benefits and harms on older persons' willingness to take medication for primary cardiovascular prevention. Arch Intern Med 2011;171:923-8.

17. Al-Rashed SA, Wright DJ, Roebuck N, et al. The value of inpatient pharmaceutical counselling to elderly patients prior to discharge. Br J Clin Pharmacol 2002;54:657-64.

18. Garfinkel D, Zur-Gil S, Ben-Israel J. The war against polypharmacy: a new cost-effective geriatric-palliative approach for improving drug therapy in disabled elderly people. Isr Med Assoc $J$ 2007;9:430-4.

19. Garfinkel D, Mangin D. Feasibility study of a systematic approach for discontinuation of multiple medications in older adults: addressing polypharmacy. Arch Intern Med 2010;170:1648-54.

20. Starner CI, Norman SA, Reynolds RG, et al. Effect of a retrospective drug utilisation review on potentially inappropriate prescribing in the elderly. Am J Geriatr Pharmacother 2009;7:11-19.

21. Graves T, Hanlon JT, Schmader KE, et al. Adverse events after discontinuing medications in elderly outpatients. Arch Intern Med 1997;157:2205-10.

22. Lindström K, Ekedahl A, Carlsten A, et al. Can selective serotonin inhibitor drugs in elderly patients in nursing homes be reduced? Scand J Prim Health Care 2007;25:3-8.

23. Gallagher PF, O'Connor MN, O'Mahony D. Prevention of potentially inappropriate prescribing for elderly patients: a randomised controlled trial using STOPP/START criteria. Clin Pharmacol Ther 2011;89:845-54.

24. Iyer S, Naganathan V, McLachlan AJ, et al. Medication withdrawal trials in people aged 65 years and older: a systematic review. Drugs Aging 2008;25:1021-31.

25. Hill KD, Wee R. Psychotropic drug-induced falls in older people A review of interventions aimed at reducing the problem. Drugs Aging 2012;29:15-30.

26. Gnjidic D, Le Couteur DG, Kouladjian L, et al. Deprescribing trials: methods to reduce polypharmacy and the impact on prescribing and clinical outcomes. Clin Geriatr Med 2012;28:237-53.

27. Kaur S, Mitchell G, Vitetta L, et al. Interventions that can reduce inappropriate prescribing in the elderly: a systematic review. Drugs Aging 2009;26:1013-28.

28. Ostini R, Jackson C, Hegney D, et al. How is medication prescribing ceased? A systematic review. Med Care 2011;49:24-36. 